\section{Determination of the TLR4 Genotype Using Allele-Specific PCR}

\author{
BioTechniques 31:___(July 2001)
}

Despite significant advances in understanding the molecular basis of sepsis and its associated immunological response, sepsis remains a problem worldwide and is associated with a high mortality. Annually, septic shock, the most severe form of sepsis, causes the death of more than 100000 people in the USA $(3,4)$. Gram-negative bacteria are the most common pathogens associated with bacterial infections in diseases such as meningitis and represent more than $50 \%$ of septic shock-related bacteria. Endotoxin or lipopolysaccharide (LPS), the main component of the cell wall of Gram-negative bacteria, has been shown to elicit an inflammatory response that mimics all of the features described in septic shock (2). Recently, we have reported the association of two common polymorphisms in the human TLR4 gene with hyporesponsiveness to inhaled LPS (1). These mutations consist of single nucleotide polymorphisms (SNPs) in the coding region of the gene, both of which result in missense mutations. Analysis of additional patients revealed an association between TLR4 genotype and susceptibility to Gram-negative infections (unpublished data). The frequency of these mutations was between $6 \%$ and $10 \%$ in all tested Caucasian populations, making this mutation a common enough polymorphism to be of diagnostic value (1). Because of the potential usefulness of the TLR4 mutation in risk-stratifying patients vulnerable to endotoxin-induced disease, we developed a quick and reliable PCR-based genotyping assay. By altering the forward PCR primers, we were able to generate mutant TLR4-specific restriction sites that allow TLR4 genotyping using a simple restriction digest following the PCR amplification of genomic patient DNA.

Study populations were made available through collaborations with several clinical facilities as indicated. For the graft-versus-host disease (GVHD) sample population, DNA samples were obtained from a previously described cohort of patients $(n=237)$ who received a hematopoietic stem cell transplantation (HSCT) from a human leukocyte antigen (HLA)-identical sibling (5). All patients received methotrexate and cyclosporine for GVHD prophylaxis and had either grade 0 or grades II-IV acute GVHD. Patients with grade I GVHD, patients with renal failure requiring dialysis, and patients without GVHD who died before day 80 after transplantation were excluded. DNA purification was done using a commercially available kit. For childhood asthma cases, we identified 120 cases with symptomatic asthma at study entry and 240 controls without persistent asthma at study entry. Subjects must have buccal cell DNA available to be eligible.

Some of the genomic DNA used was derived from whole blood and purified using commercially available kits, such as Gentra Systems ${ }^{\mathrm{TM}}$ (Minneapolis, MN, USA). DNA purified using kits works very well in this assay. DNA purified as crude lysate using the $\mathrm{NaOH}$ lysis method will work as well, although, in general, a larger amount of sample is required to yield sufficient amounts of PCR product.

PCR primers were designed to allow a distinction of wild-type and mutant TLR4 alleles based on the presence of restriction enzyme recognition sites. In both cases, the forward primer sequences were altered to generate a restriction site in the mutant allele. The following primers were used: for TLR4 Asp299Gly, forward 5'-GATTAGCATACTTAGACTACTACCTCEAATG-3'

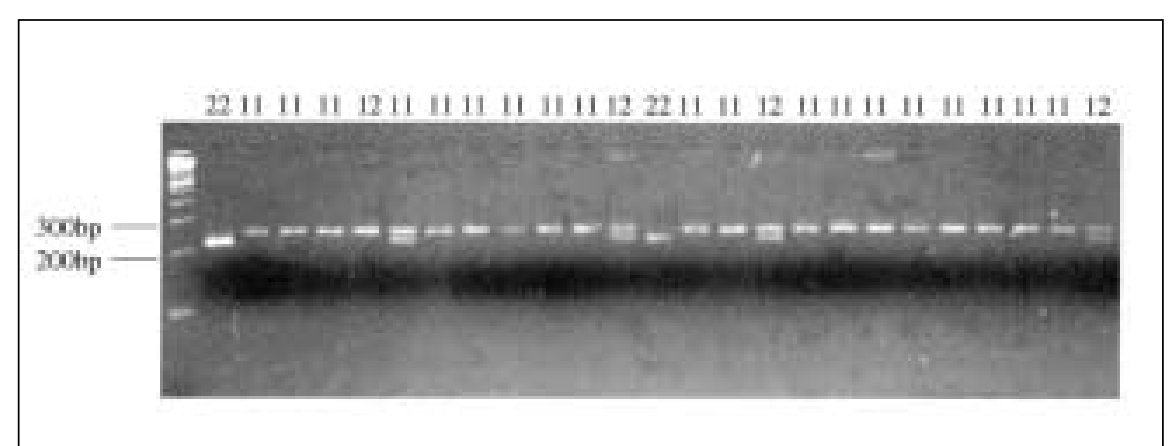

Figure 1. Gel electrophoresis of NcoI digest for TLR4 Asp299Gly RFLP assay. Overnight digest (10 $\mu \mathrm{L}$ ) was mixed with $2 \mu \mathrm{L}$ loading buffer. A 100-bp ladder (Life Technologies, Rockville, MD, USA) was included on the left as a size standard. Genotype assignments are indicated above each lane (1 for wildtype allele, 2 for mutant allele). AAAAGCATTCCCAC-3', and for TLR4 Thr399Ile, forward 5'-GGTTGCTGTTCTCAAAGTGATTTTGGGAGAA-3' and reverse 5'-ACCTGAAGACTGGAGAGTGAGTTAAATGCT$3^{\prime}$. The underlined bases in both forward primers indicate the nucleotide altered to create a NcoI (TLR4 Asp299Gly) and HinfI (TLR4 Thr399Ile) restriction site, respectively.

Reactions are set up using the Ampli$\mathrm{Taq}^{\circledR}$ PCR kit (Applied Biosystems, Foster City, CA, USA). Other PCR kits, such as the Advantage ${ }^{\mathrm{TM}}$ cDNA-PCR kit (Clontech Laboratories, Palo Alto, CA, USA) will work equally well. In a total reaction volume of $25 \mu \mathrm{L}, 2.5 \mu \mathrm{L} 10 \times$ PCR buffer, 20 pmol each primer (see above), $0.02 \mu \mathrm{g}(0.2 \mu \mathrm{g}$ for crude lysate) genomic DNA, 5 U Taq DNA polymerase, and $1 \mu \mathrm{L}$ dNTP mixture (Clontech Laboratories) are combined. Reactions are run on a MJ Tetrad ${ }^{\mathrm{TM}}$ PTC225 Thermo Cycler (MJ Research, Waltham, MA, USA) using the following conditions: $95^{\circ} \mathrm{C}$ for $4 \mathrm{~min}$, then 30 cycles of $95^{\circ} \mathrm{C}$ for $30 \mathrm{~s}, 55^{\circ} \mathrm{C}$ for $30 \mathrm{~s}$, and $72^{\circ} \mathrm{C}$ for $30 \mathrm{~s}$ ). Four microliters of the resulting PCR products are used for an overnight digest with the appropriate restriction enzyme, and digests are run out on a $3 \%$ NuSieve ${ }^{\circledR}$ GTG agarose gel (BMA, Rockland, ME, USA) to determine the TLR4 alleles.

For the purpose of validating this method, 10 patient samples with different genotypes were sequenced directly using an ABI 377 sequencer (Applied Biosystems). Genomic DNA was amplified using forward 5'-TCTAGAGGGCCTGTGCAATT-3' and reverse and reverse 5'-GATCAACTTCTGA- 


\section{5'-TGAAAACTCACTCATTTGTTT-} CAA-3' primers. The amplified region of genomic DNA contains both the 299 and 399 residues, and the complete TLR4 genotype can be assayed in one sequencing run.

We tested the RFLP assay by screening several patients of unknown genotype. Twenty nanograms genomic DNA were amplified using standard reagents in a $25-\mu \mathrm{L}$ PCR volume to ensure that the PCR was robust and reproducible. Twenty nanograms of genomic DNA are the regular template amount used in
PCR in the laboratory to amplify human genomic DNA for genotyping purposes. We used two different kits, the Advantage cDNA-PCR kit and the AmpliTaq PCR kit. Both reagent kits produced equal results in terms of amount of amplified product and specificity of the amplification reaction. Following the amplification step, the PCR product for the TLR4 Asp299Gly-specific mutation was digested using $\mathrm{NcoI}$ (New England Biolabs, Beverly, MA, USA). This enzyme is not sensitive to salt concentrations, nor does it require special incuba-

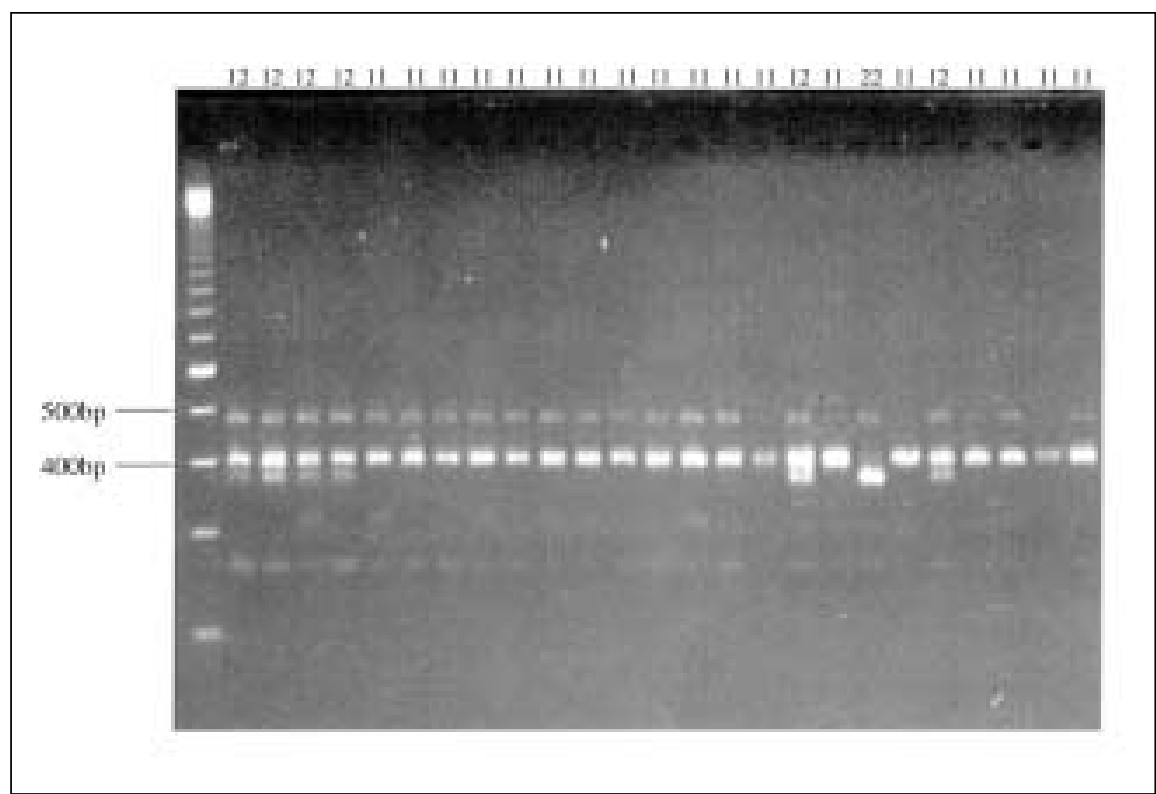

Figure 2. Gel electrophoresis of HinfI digest for TLR4 Thr399Ile RFLP assay. Overnight digest (10 $\mu \mathrm{L}$ ) was mixed with $2 \mu \mathrm{L}$ loading buffer. A 100-bp ladder was included on the left as a size standard. Genotype assignments are indicated above each lane (1 for wild-type allele, 2 for mutant allele).

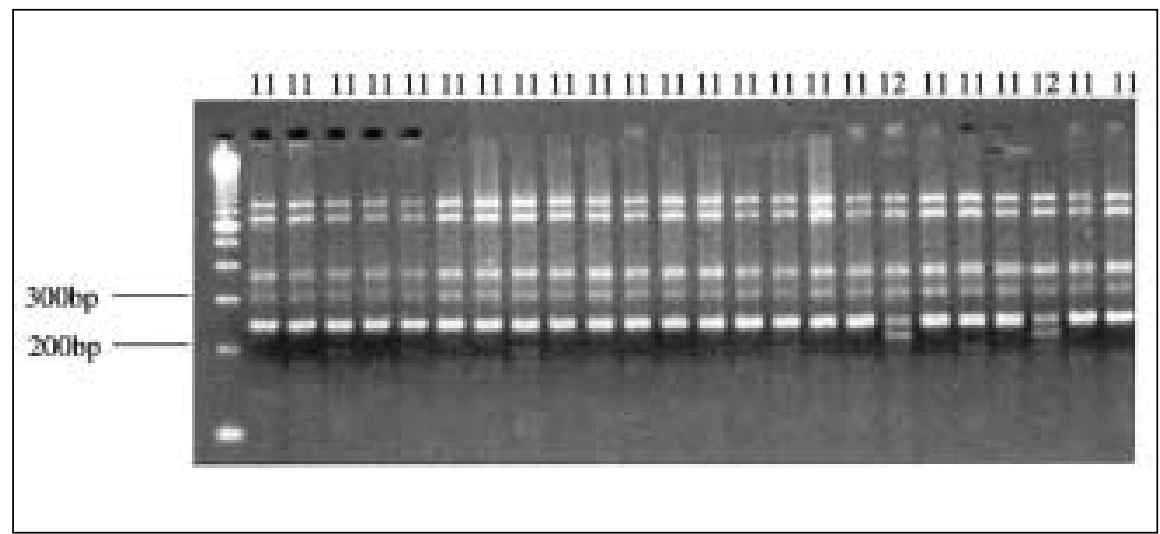

Figure 3. Gel electrophoresis of NcoI digest for TLR4 Asp299Gly RFLP assay. DNA was a crude prep of buccal swabs. Extra bands caused by nonspecific annealing during the PCR amplification are visible. Overnight digest $(10 \mu \mathrm{L})$ was mixed with $2 \mu \mathrm{L}$ loading buffer. A 100-bp ladder was included on the left as a size standard. Genotype assignments are indicated above each lane ( 1 for wild-type allele, 2 for mutant allele).

tion conditions. We routinely digest 4 $\mu \mathrm{L}$ of a $25-\mu \mathrm{L}$ PCR overnight. The PCR product does not have to be purified, but oil carryover into the enzyme digest tube should be avoided. The digest reaction is set up using $4 \mu \mathrm{L}$ PCR product, 1 $\mu \mathrm{L} 10 \times$ enzyme buffer (New England Biolabs; buffer no. 2), $0.5 \mu \mathrm{L} \mathrm{NcoI} \mathrm{en-}$ zyme, and $4.5 \mu \mathrm{L}$ water. The digest is placed in a humidified $\mathrm{CO}_{2}$ incubator overnight at $37^{\circ} \mathrm{C}$ to prevent evaporation. The following day, five microliters of the reaction are run on a $3 \%$ NuSieve gel to determine the TLR4 alleles at residue 299 (Figure 1). The PCR and subsequent digest for the genotyping of TLR4 residue 399 is done accordingly, with the exception of using HinfI (New England Biolabs) enzyme for the digest (Figure 2). Both digestions give two clear bands in the heterozygote and single bands for both homozygous mutant and wild-type patients.

This assay also works with crude buccal DNA preps. Figure 3 shows the gel for a TLR4 Asp299Gly assay using $0.2 \mu \mathrm{g}$ crude DNA from buccal swabs. As expected, the low quality of the DNA causes additional bands in the PCR but does not interfere with the subsequent enzyme digestions. Genotyping of low-quality DNA is still possible using this method.

Ten patient samples from the original healthy control population were sequenced as described above, and the genotype assignments confirmed the results of the PCR-based RFLP assay. This demonstrates that the RFLP assay described in this paper allows a quick and reliable way to genotype patients for the TLR4 Asp299Gly and Thr399Ile mutations (data not shown).

To determine whether the TLR4 polymorphisms described previously can be used as genetic indicators of severity in microbial diseases, we screened a variety of patient and control populations. We wanted to ensure that the allele frequency of the TLR4 mutations was sufficiently high to test for these mutations routinely in patients with infectious diseases, such as sepsis, or patients suffering from diseases that are complicated by recurrent bacterial infections. A screen of more than 2000 Caucasians, consisting of both healthy controls and patients, revealed that the allele frequency for both the TLR4 
Asp299Gly and the TLR4 Thr399Ile alleles was at least $6 \%$ in all populations tested. This frequency is sufficiently high to screen at-risk patients routinely for these mutations.

In summary, we have developed a quick and reliable assay to determine the genotype of patients with respect to two common polymorphisms in the TLR4 receptor. The assay described in this paper allows the differentiation between patients who carry mutations at both loci and those who carry only one of the mutations. This discrimination may be important because there is an indication that the two mutations are of different severity and may confer a different amount of risk to the carrier. The simple PCR-based approach allows this genotyping assay to be performed in any laboratory with no need for expensive, specialized equipment. The assay uses standard molecular biology tools and common laboratory equipment and can therefore be performed in most clinical laboratories.

\section{REFERENCES}

1.Arbour, N.A., E. Lorenz, B.C. Schutte, J. Zabner, J.N. Kline, M. Jones, K.L. Frees, J.L. Watt et al. 2000. TLR4 mutations are associated with endotoxin hyporesponsiveness in humans. Nat. Genet. 25:187-191.

2.Bone, R.C. 1991. Gram-negative sepsis: background, clinical features, and intervention. Chest 100:802-808.

3.Mendez, C., A.A. Kramer, K.F. Salhab, G.A. Valdes, J.G. Norman, K.J. Tracey, and L.C. Carey. 1998. Tolerance to shock: an exploration of mechanism. Ann. Surg. 229:843-850.

4.Tracey, K.J. and E. Abraham. 1999. From mouse to man: or what have we learned about cytokine-based anti-inflammatory therapies? Shock 11:224-225.

5.Tseng, L.H., M.T. Lin, J.A. Hansen, T. Gooley, J. Pei, A.G. Smith, E.G. Martin, E.W. Petersdorf et al. 1999. Correlation between disparity for the minor histocompatibility antigen HA-1 and the development of acute graftversus-host disease after allogeneic marrow transplantation. Blood 94:2911-2914.

Address correspondence to Dr. Eva Lorenz, Department of Medicine, Section of Infectious Diseases, Wake Forest Medical Center, Medical Center Blvd., WinstonSalem, NC 27157-1042, USA. e-mail: elorenz@wfubmc.edu

Received 26 December 2000; accepted 17 April 2001.

Eva Lorenz, Kathy L. Frees, and David A. Schwartz Duke University Medical Center

Durham, NC, USA

M13-Tailed Primers Improve the Readability and Usability of Microsatellite Analyses Performed with Two Different AlleleSizing Methods

BioTechniques 31:____(July 2001)

Microsatellite analysis, also referred to as simple sequence length polymorphism (SSLP) or short tandem repeat polymorphism (STRP), is a widely used technique for genome-wide mapping projects. As the number of primer pairs that become available for an evergreater variety of organisms increases, the usage and applicability of this tool grow. The principle of the technique is to use primer pairs that flank repetitive DNA sequences to amplify samples of genomic DNA and to examine the size of the amplified alleles on a sequencing device. Despite the increasing availability of automatic sequencers in research facilities, a large majority of laboratories still analyze microsatellites "manually". This involves labeling one of the two PCR primers (usually the forward one) with [32P] $\gamma$-ATP and further analysis of the amplified alleles on acrylamide sequencing gels. The use of an automatic sequencer might greatly increase genotyping output. However, such sequencers require labeling one of the primers of each pair with a dye that is compatible with its laser recognition system. Given the cost of fluorescently labeled primers, it becomes prohibitively expensive to use an automatic sequencer for a project that involves hundreds of markers.

One possible alternative is M13tailed primers, where every forward primer is $5^{\prime}$-tailed with the M13 sequence 5'-CACGACGTTGTAAAACGAC-3' (2,3). The M13-forward primers are used in combination with an M13 primer that has the same sequence but is dye-labeled at its $5^{\prime}$ end. The latter primer is the sole source of label and can be used with any M13forward primer to generate a labeled amplified allele. M13-tailed primers, which can be obtained inexpensively either from vendors or by custom synthesis, have previously been shown to amplify infrared dye-labeled alleles that can be analyzed on an $\mathrm{IR}^{2}$ Automated DNA sequencer (LI-COR, Lincoln, NE, USA) automatic sequencer (2). However, it has not been shown how the pattern of alleles amplified with M13 primers compared to that of untailed primers or whether the technique was applicable to dyes used with other automatic sequencers.

To answer these questions, we first compared the patterns of alleles amplified with primers (with or without M13 tailing) end-labeled with $\left.{ }^{32} \mathrm{P}\right] \gamma$-ATP 